\title{
Escitalopram y extrasístoles ventriculares
}

\author{
Juan José Criado-Álvareza, , Jaime González González,o, \\ Silvia Correa Martín ${ }^{a}$ y Carmen Romo Barrientos ${ }^{d}$.
}

\begin{abstract}
a Centro de Salud de La Pueblanueva. Gerencia de Atención Integrada de Talavera de la Reina. Servicio de Salud de Castilla-La Mancha (SESCAM). España. b Departamento de Medicina. Facultad de Terapia Ocupacional, Logopedia y Enfermería. Universidad de Castilla-La Mancha (España). c Centro de Salud de Santa Olalla. Gerencia de Atención Integrada de Talavera de la Reina. SESCAM (España). ¿ Servicio de Salud Mental. Hospital Nuestra Señora del Prado. Gerencia de Atención Integrada de Talavera de la Reina. SESCAM (España).
\end{abstract}

Correspondencia: Juan José Criado-Álvarez. C/ Mateo Ramos de la Nava, 7. C.P. 45654. San Bartolomé de las Abiertas - Toledo (España).

Correo electrónico: jjcriado@sescam.jccm.es

Recibido el 23 de agosto de 2014.

Aceptado para su publicación el 15 de noviembre de 2014.

\begin{abstract}
RESUMEN
La toxicidad cardíaca de los inhibidores selectivos de la recaptación de serotonina como citalopram y escitalopram ha sido asociada a un alargamiento del intervalo QT en el electrocardiograma. Presentamos el caso de extrasístoles ventriculares bigeminados asociados al consumo de escitalopram. Deberíamos evitar su uso en pacientes con historia personal o familiar de muerte súbita, sexo femenino, insuficiencia cardíaca congestiva descompensada, infarto de miocardio, bradiarritmias, alteraciones iónicas, y antecedentes de intervalo QT alargado, además de monitorizar electrocardiográficamente a los pacientes mayores de 65 años.
\end{abstract}

Palabras Clave. Antidepresivos. Citalopram. Extrasístole Ventricular. Síndrome de QT prolongado.

\begin{abstract}
Escitalopram and ventricular extrasystoles

Cardiac toxicity of selective serotonin reuptake inhibitors, such as citalopram and escitalopram, has been associated with a prolonged QT interval on the electrocardiogram. We present a case of bigeminal ventricular extrasystole associated with escitalopram use, which should be avoided in patients with a personal or family history of sudden death, female patients, decompensated congestive heart failure, myocardial infarction, bradyarrhythmias, electrolyte changes, and a history of long QT interval. In patients over 65, electrocardiographic monitoring is also advisable.
\end{abstract}

Key words: Antidepressive Agents. Citalopram. Ventricular Extrasystole. Long QT Syndrome.

\section{INTRODUCCIÓN}

Las agencias reguladoras de medicamentos de Europa y de Estados Unidos han alertado sobre la posible toxicidad cardíaca de los antidepresivos tricíclicos y los inhibidores selectivos de la recaptación de serotonina como citalopram y escitalopram, relacionándolos con un alargamiento del intervalo QT en el electrocardiograma, extrasístoles ventriculares (EV) y, posteriormente, con el riesgo de agravar o provocar una taquicardia ventricular polimórfica denominada "torsade de pointes" (TdP) ${ }^{1}$. Habitualmente se resuelve espontáneamente, aunque en algunos casos produce una fibrilación ventricular y pudiera asociarse a muerte súbita cardíaca. La probabilidad de la misma y de otras arritmias se incrementa en pacientes de edad avanzada, y ante la presencia de comorbilidades y alteraciones hepáticas o renale ${ }^{2-4}$. Los EV son una taquiarritmia ventricular que se produce por una despolarización precoz del miocardio ventricular, con una etiología de base estructural (enfermedad coronaria, miocardiopatía o valvulopatía), consumo de estimulantes (café, alcohol), fármacos (digital, teofilina, antidepresivos tricíclicos...) o idiopática, presentándose en la clínica con presíncopes, palpitaciones y mareos, y en el electrocardiograma con un complejo QRS precoz, ancho y deformado, sin onda $P$ reconocible que lo preceda ${ }^{5,6}$. Se presenta el caso de una paciente con EV asociado al consumo de escitalopram. 


\section{OBSERVACIONES CLÍNICAS}

Es una mujer de 80 años, sin historia familiar de cardiopatía ni de hábitos tóxicos; como factores de riesgo presenta obesidad moderada, dislipemia tratada con simvastatina $20 \mathrm{mg} / 24 \mathrm{~h}$ e hipertensión arterial controlada con enalapril $20 \mathrm{mg} / 24 \mathrm{~h}$. En sus antecedentes personales existe una pancreatitis aguda biliar y posterior colecistectomía, litiasis renal, megacolon, hipotiroidismo primario controlado (TSH: 1,33 mU / I) en tratamiento con levotiroxina sódica de $88 \mu \mathrm{g} / 24 \mathrm{~h}$, y una valvulopatía mitroaórtica sin repercusión hemodinámica que es vigilada desde el Servicio de Cardiología. Desde el año 2009 ha sido tratada en el Servicio de Salud Mental con diferentes ansiolíticos y antidepresivos por una depresión neurótica. Desde el mes de octubre de 2013 es tratada con escitalopram $10 \mathrm{mg} / 24 \mathrm{~h}$ y zolpidem $10 \mathrm{mg} / 24 \mathrm{~h}$. En el mes de mayo de 2014 presenta de forma esporádica un cuadro clínico con palpitaciones, leve mareo y nerviosismo, que es autolimitado y se resuelve sin tratamiento. La exploración es anodina salvo un soplo mesosistólico en región mitroaórtica. Estos síntomas se incrementan en el mes de junio de 2014, por lo que se realiza una analítica que es normal y un electrocardiograma que presenta un ritmo sinusal a 60 latidos por minuto, con complejos
QRS anchos monomórficos en bigeminismo (cada complejo normal es seguido de un doblete), cuyo diagnóstico es confirmado por el Servicio de Cardiología como EV monomorfos bigeminados con buena tolerancia clínica (figura 1). Se informa a la paciente de que su valvulopatía y arritmia no precisan tratamiento en el momento actual, excepto si presentase síntomas o inestabilidad hemodinámica. La paciente está a la espera de nuevos estudios cardiológicos en atención hospitalaria. Ante la sospecha de que el escitalopram sea el causante de la arritmia se decide su retirada progresiva y paulatina, realizando tres electrocardiogramas de control (todos ellos normales) en el plazo de dos meses desde el inicio de los síntomas (figura 2). Los EV han desaparecido tras la primera semana sin escitalopram y la paciente no refiere síntomas o clínica acompañante de tipo cardiológico o relacionada con salud mental. El médico de atención primaria sustituyó el escitalopram por fluoxetina 20 mg / día para el tratamiento de la depresión neurótica, y ha remitido a la paciente a Salud Mental para una revisión, aunque está asintomática en el momento actual. El cuadro ha sido declarado como efecto adverso en la historia clínica electrónica y se solicitó el consentimiento verbal a la paciente para su publicación.

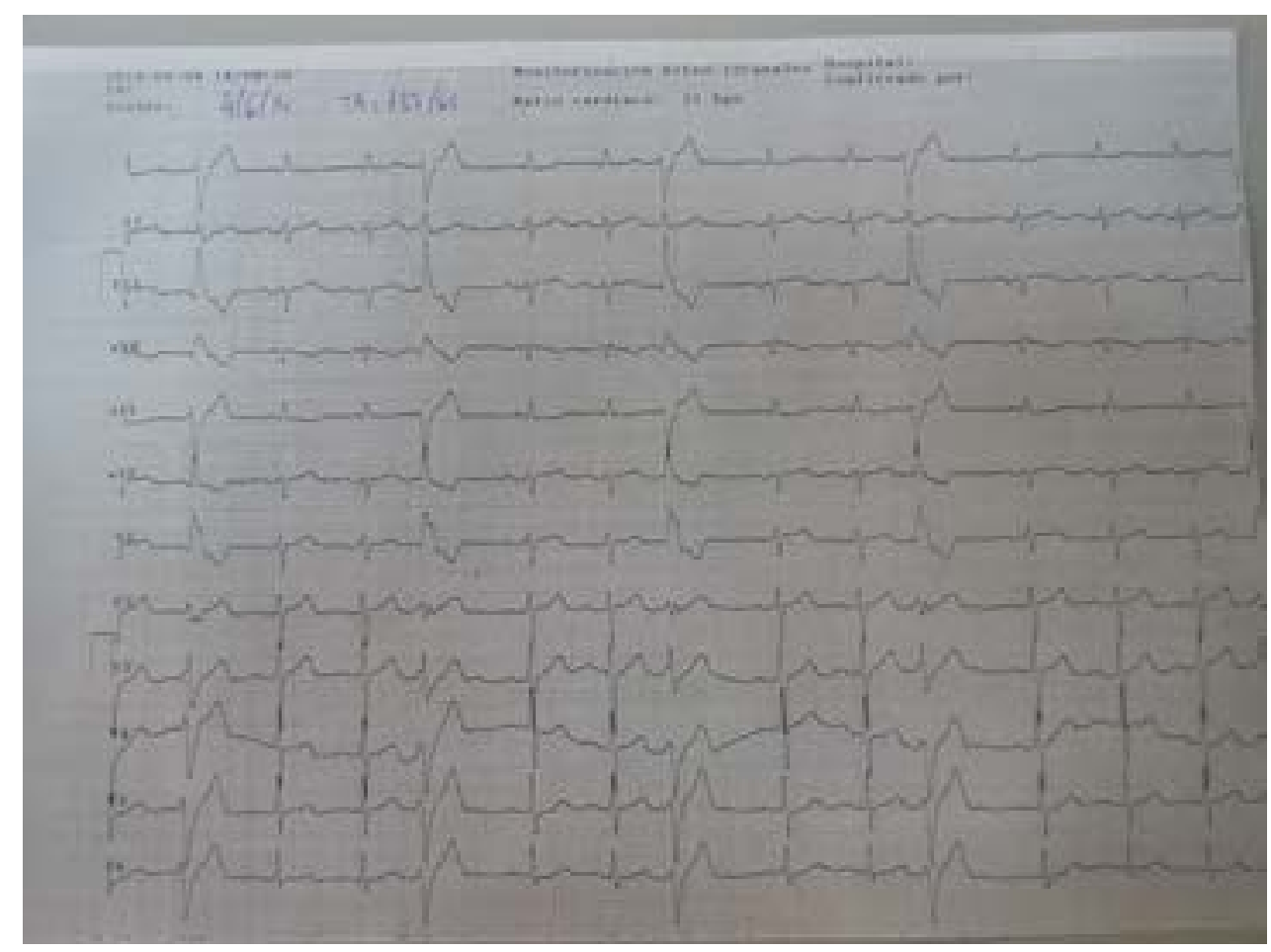

Figura 1. Electrocardiograma con extrasístoles ventriculares bigeminados 


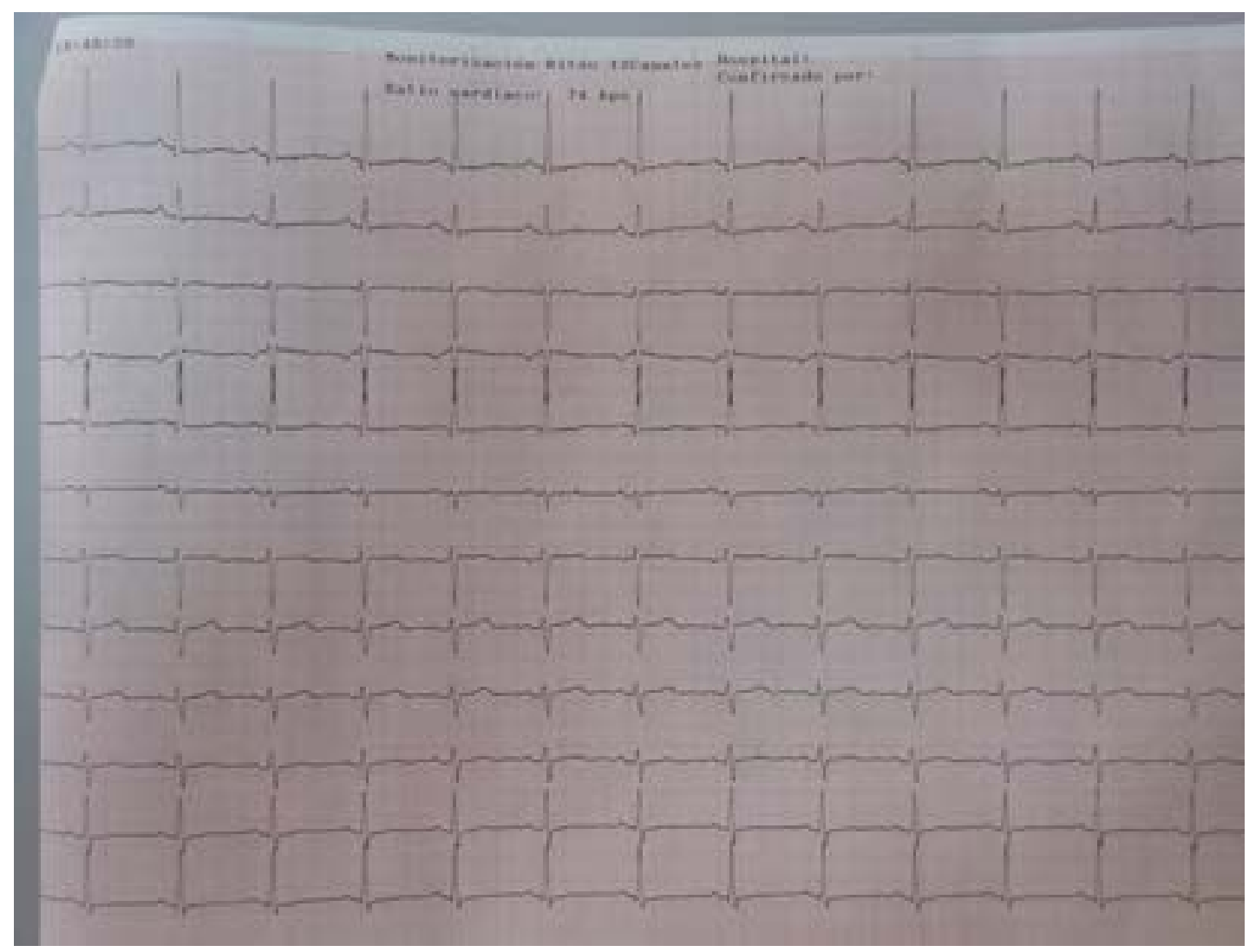

Figura 2. Electrocardiograma un mes después de suspender escitalopram

\section{DISCUSIÓN}

El consumo de antidepresivos para el tratamiento de trastornos mentales comunes se ha incrementado en las últimas décadas. En España, en el año 2012 el 7 \% de la población refería haber tomado un antidepresivo en las dos semanas anteriores, duplicándose ese porcentaje en las personas mayores. El consumo de inhibidores selectivos de la recaptación de la serotonina se ha incrementado desde 2000 a 2010 en un 63,9 \%, pero en el caso del escitalopram tiene un incremento desde su aparición en 2004 del $1013 \%$, suponiendo el $28,4 \%$ de la cuota de mercado y desplazando a la paroxetina ${ }^{7}$. La generalización en el uso de estos fármacos hace que debamos ser prudentes en su prescripción ${ }^{1}$. Según diferentes estudios parece que el citalopram y el escitalopram son razonablemente seguros ${ }^{4,8,9}$; sin embargo, deberíamos tener una serie de precauciones como evitar su uso en pacientes con historia personal o familiar de muerte súbita, sexo femenino, insuficiencia cardíaca congestiva descompensada, infarto de miocardio, bradiarritmias, alteraciones iónicas (hipocaliemia, hipomagnesemia, hipocalcemia), y antecedentes de intervalo QT alargado $2,8,9$. Se debería monitorizar electrocardiográficamente a los pacientes, especialmente los mayores de 65 años, no debiendo superar la dosis máxima diaria de $10 \mathrm{mg}$ en el caso del escitalopram ${ }^{1,10}$. En caso de sustitución del citalopram o del escitalopram ante la aparición de efectos adversos, el nuevo fármaco debe ser monitorizado con electrocardiogramas periódicos ${ }^{2,10}$. La American Medical Association (http://www.azcert.org/medical-pros/drug-lists/drug-lists.cfm) y el Arizona Center for Education and Research on Therapeutics (www.qtdrugs.org) mantienen al día listados por grupos de riesgo. Ofrecen información de los fármacos que potencialmente pueden prolongar el intervalo QT y aumentar el riesgo de sufrir $\operatorname{TdP}^{2,10}$. Según el grado de asociación causal entre el fármaco y el efecto adverso se clasifica a los fármacos en asociación definida (citalopram y escitalopram), posible (mirtazipina, venlafaxina) y condicionada (amitriptilina, clomipramina, fluoxetina, paroxetina, sertralina, trazodona), donde la evidencia sostiene que pueden prolongar el intervalo QT solo bajo determinadas condiciones de sobredosis o interacciones medicamentosas ${ }^{2}$. 
Las arritmias ventriculares tienen una presentación en el $75 \%$ de los casos en forma de EV monomorfos, seguido de casos de EV y taquicardias ventriculares no sostenidas repetitivas, con un pronóstico en general benigno ${ }^{5,6}$. El caso clínico descrito corresponde a una probable asociación entre el consumo de escitalopram y su potencial arritmogénico, que puede ser detectado y manejado fácilmente por el médico de atención primaria cuando tiene una buena coordinación con la atención hospitalaria.

\section{BIBLIOGRAFÍA}

1. Agencia Española de Medicamentos y Productos Sanitarios (AEMPS) [sede web]. Madrid: AEMPS; 02/12/2011. Escitalopram: Prolongación del intervalo QT del electrocardiograma (Nota Informativa MUH (FV) 23/2011). Disponible en: http://www.aemps.gob.es/informa/notasInformativas/ medicamentosUsoHumano/seguridad/2011/docs/NIMUH_23-2011.pdf

2. Villamañán $\mathrm{E}$, Armada $\mathrm{E}$, Ruano $\mathrm{M}$. Prolongación del intervalo QT inducido por fármacos: ¿conocemos sus riesgos? Med Clin (Barc). 2014. DOI: 10.1016/j.medcli.2014.01.027

3. Roden DM. Drug-induced prolongation of the QT Interval. N Engl J Med. 2004; 350 (10):1013-22.

4. Castro VM, Clements CC, Murphy SN, Gainer VS, Fava M, Weilburg JB et al. QT interval and antidepressant use: a cross sectional study of electronic health records. BMJ. 2013; 346:f288. DOI: 10.1136/bmj.f288

5. Baquero Alonso M, Rodríguez Martín AM, González Carnero R, Gómez Santana JC, de Haro Muñoz J. Recomendaciones de buena práctica clínica en arritmias. SEMERGEN. 2010; 36 (1): 31-43. DOI: 10.1016/j.semerg.2009.09.004

6. Arias MA, Pachón M, Casares-Medrano J, Puchol A. Arritmias ventriculares en bigeminismo continuo: una forma atípica de arritmias idiopáticas. Rev Clin Esp. 2012; 212 (6): 321-3. DOI: 10.1016/j.rce.2012.01.004

7. Sempere Verdú E, Salazar Fraile J, Palop Larrea V, Vicens Caldentey C. Evolución de la utilización de antidepresivos, ansiolíticos e hipnóticos en la Comunitat Valenciana. Período 2000-2010. Aten Primaria. 2014; 46 (8):416-25. DOI: 10.1016/j.aprim.2013.11.006

8. Beach SR, Kostis WJ, Celano CM, Januzzi JL, Ruskin JN, Noseworthy PA et al. Meta-analysis of selective serotonin reuptake inhibitor-associated QTc prolongation. J Clin Psychiatry. 2014; 75 (5): 441-9. DOI 10.4088/jcp.13r08672

9. Leonard CE, Bilker WB, Newcomb C, Kimmel SE, Hennessy S. Antidepressants and the risk of sudden cardiac death and ventricular arrhythmia. Pharmacoepidemiol Drug Saf. 2011; 20 (9): 903-13. DOI: 10.1002/pds.2181

10. Álvarez E, Vieira S, Garcia-Moll X. Citalopram, escitalopram y QT largo: ¿alerta o alarma? Rev Psiquiatr Salud Ment. 2014; 7 (3): 147-50. DOI 10.1016/j.rpsm.2013.12.005 